\title{
Modelado y control de un sistema de levitación magnética
}

\section{Model and control of magnetic levitation system}

\author{
Diego A. Bravo M. $*_{*}$ (D), Carlos F. Rengifo R.*(D), Luis F. Moncayo \\ M. ${ }^{+}$ \\ + Departamento de Física, Universidad del Cauca, Popayán, Cauca, \\ Colombia \\ * Departamento de Electrónica, Instrumentación y Control, \\ Universidad del Cauca, Popayán, Cauca, Colombia
}

\begin{abstract}
Resumen. En este artículo se realizó el modelado y control de un sistema de levitación magnética ( MAGLEV). Para ello se construyó un prototipo con materiales de bajo costo con el objetivo de realizar prácticas experimentales para la enseñanza de sistemas de control en el programa de Ingeniería Física. Los resultados muestran que es posible utilizar el sistema para controlar la posición de una bola mediante una estrategia de control lineal.
\end{abstract}

Palabras Claves. Modelo Dinámico; sistemas de control; levitación magnética.

\begin{abstract}
In this article, the modeling and control of a magnetic levitation system (MAGLEV) was carried out. For this, a prototype with low-cost materials was built with the aim of carrying out experimental practices for the teaching of control systems in the Physical Engineering program. The results show that it is possible to use the system to control the position of a ball using a linear control strategy.
\end{abstract}

Keywords. Dynamic model; control systems; magnetic levitation.

Como Citar. D. A. Bravo M., C. F. Rengifo R. y L. F. Moncayo M., "Model and Control of Magnetic Levitation System", Jou. Cie. Ing., vol. 13, no. 1, pp. 1-12, 2021. doi $10.46571 / \mathrm{JCl} .2021 .1 .1$

Recibido: 23/09/2020 Revisado: 02/02/2021 Aceptado: 17/02/2021

\section{Introduccción}

Desde 1971, cuando en la ciudad de Ottobrun-Alemania, se presentó la primera versión de "Transrapid" por la empresa Messerschmitt-Böolkow-Blohm, la levitación magnética se convirtió en un foco de interés para los países que quisieron implementar dicha tecnología [1, 2]. Debido a los altos costos que se requerían para la construcción de nuevas líneas de tren especiales, se requirió realizar investigaciones sobre posibles alternativas, que llevó al desarrollo de nuevas tecnologías en muchos campos, desde nuevos tipos de amplificadores, transductores, hasta nuevas 
geometrías de electroimanes que les permitieran hacer cambio de rutas. Las estrategias de control también tuvieron que ser adaptadas a dichas tecnologías emergentes [3, 4].

El interés por la levitación magnética llevó a que científicos alrededor del mundo desarrollaran prototipos del sistema MAGLEV para su estudio en control, especialmente en países como China, Japón, Alemania y el Reino Unido [5, 6, 4. Al pasar de los estudios, la planta MAGLEV se convirtió en un desafío para la ingeniería debido a su dinámica no lineal altamente inestable, provocando que se construyeran plantas de experimentación en muchas universidades. En la actualidad MAGLEV es reconocida como una planta de experimentación clásica en los sistemas de control [7].

Un sistema de levitación magnética es de gran interés para ilustrar varios conceptos en física e ingeniería [8]. Este sistema es no lineal e inestable [9]. Tales particularidades han incrementado el interés de muchos investigadores en proporcionar herramientas de enseñanza e investigación que permitan afianzar los conocimientos relacionados con las diferentes estrategias de control [10.

La planta para la experimentación MAGLEV, consiste en un cuerpo que se suspende en el aire mediante el uso de campos magnéticos. Los componentes principales son: Un electroimán (EI), una bola de acero, un pedestal que sostiene todos los demás componentes y un sensor que mida la distancia entre la bola y la superficie inferior del EI. El objetivo de control es estabilizar la posición de la bola de acero en un punto bajo el electroimán, haciendo un control de la corriente que pasa a través de él, por medio de la variación del voltaje aplicado a este.

En este artículo se describe el modelado y control de una planta MAGLEV que sirve para realizar prácticas experimentales en el área de sistemas de control. Se propuso que la planta fuera de bajo costo y robusta. Para controlar la planta MAGLEV, el estudiante debe seguir los pasos descritos a continuación. Primero, debe efectuar un modelado del sistema dinámico mediante ecuaciones diferenciales obtenidas mediante las leyes de la física. Las ecuaciones diferenciales que modelan el comportamiento del sistema son no-lineales, por lo que le es requerido aplicar algún método de linealización [11. Posteriormente, debe calibrar el sensor de posición, ya que se podría ver afectado por las condiciones ambientales. Para garantizar que la corriente que pasa por el EI sea la necesaria, debe implementar un controlador en el que se manipule el voltaje aplicado al EI. Finalmente se diseña y se implementa una estrategia de control lineal PID para el control de posición de la bola.

La educación en ingeniería de control exige que el estudiante se enfrente a problemas reales en la implementación de controladores, tales como el ruido de sensado, los disturbios externos y las restricciones en las variables de entrada y/o salida. Además, el estudiante tiene la oportunidad de probar diferentes estrategias de control y de realizar experimentos de identificación de sistemas reales. Las plantas didácticas son invaluables para la enseñanza de sistemas de control 7 .

El artículo esta estructurado de la siguiente manera: la sección 2 describe el modelo matemático del sistema de levitación magnética, la sección 3 está dedicada a la estrategia de control, seguido por los resultados en la sección 4 y finalizando con las conclusiones del artículo en la sección 5 .

\section{Modelo Dinámico del Sistema de Levitación Magnética}

La Figura 1 muestra un esquema del sistema de levitación magnética (MAGLEV), en el que una esfera metálica levita con la ayuda de un EI y un sensor óptico para la medición de la posición de la esfera.

A partir de la segunda ley de Newton [12], se obtiene la ecuación de movimiento que representa la dinámica del sistema.

$$
m \ddot{y}(t)=m g-\beta \dot{y}(t)+F(y, i)
$$

Donde $m$ es la masa de la esfera, $y \geq 0$ es la posición vertical (hacia abajo) de la esfera medida desde un punto de referencia ( $y=0$ cuando la esfera está pegada al EI), $\beta$ es el coeficiente de 

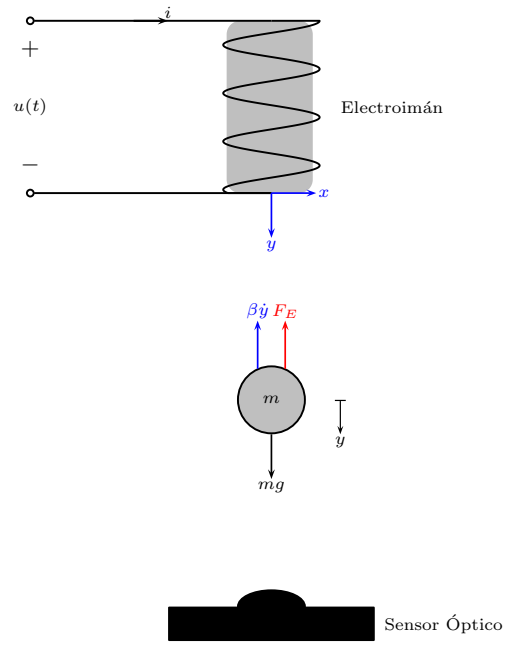

Figura 1: Planta de levitación magnética

fricción viscosa, g es la aceleración de la gravedad, $F(y, i)$ es la fuerza generada por el EI, e $i$ es la corriente que atraviesa el EI. La inductancia del EI depende de la posición de la esfera, y puede modelarse como [5]:

$$
L(y)=L_{1}+\frac{L_{0} X_{0}}{y}=L_{1}+\frac{2 c}{y}
$$

Donde:

$$
c=\frac{L_{0} X_{0}}{2}
$$

Aquí, $L_{1}$ es la inductancia de la bobina cuando la esfera no esta cerca $y=\infty, L(y)$ es una función no lineal y representa inductancia adicional ocasionada por la esfera y $c$ es un parámetro determinado por la posición del balín, $X_{0}$ es la posición de referencia. La Ec. (2) tiene su máximo valor cuando la esfera está cerca del EI y decrece a un valor constante a medida que la esfera se aleja.

La energía del campo magnético está definida como:

$$
E(y, i)=\frac{1}{2} L(y) i^{2}
$$

Entonces la fuerza $F(y, i)$ generada por el campo magnético al balín está dada por:

$$
F(y, i)=-\frac{\partial E}{\partial y}=\frac{c i^{2}}{y^{2}}
$$

El electroimán se modela como un circuito serie $R L$, utilizando la ley de voltajes de Kirchhoff:

$$
G(u)=R i(t)+\dot{\phi}(t)
$$

$\dot{\phi}(t)$ es el flujo magnético producido por el EI y G(u) es el voltaje efectivo del circuito RL, causado por la etapa de amplificación.

$$
\dot{\phi}(t)=L \frac{d i}{d t}+i \frac{d L}{d t}
$$


Usando $x_{1}=y(t), x_{2}=\dot{y}(t)$ y $x_{3}=i(t)$ como variables de estado, y $u$ como entrada de control se tiene la ecuación de estado.

$$
\begin{aligned}
& \dot{x}_{1}=x_{2} \\
& \dot{x}_{2}=g-\frac{\beta}{m} x_{2}-\frac{c x_{3}^{2}}{m x_{1}^{2}} \\
& \dot{x}_{3}=\frac{1}{L\left(x_{1}\right)}\left[-R x_{3}-\frac{2 c x_{2} x_{3}}{x_{1}^{2}}+G(u)\right]
\end{aligned}
$$

Los parámetros del sistema se obtuvieron algunos mediante instrumentos de laboratorio. Para la medición de la resistencia e inductancia se utilizó un multímetro, la masa del balín (esfera) fue medida con una balanza y su radio con un calibrador. Todos los instrumentos fueron previamente calibrados. El valor del parámetro $c$, se determina a partir de la ecuación (8) tomando $\dot{x}_{2}=0 \mathrm{y}$ donde se conoce $m, g x_{3}$ y $x_{1}$.

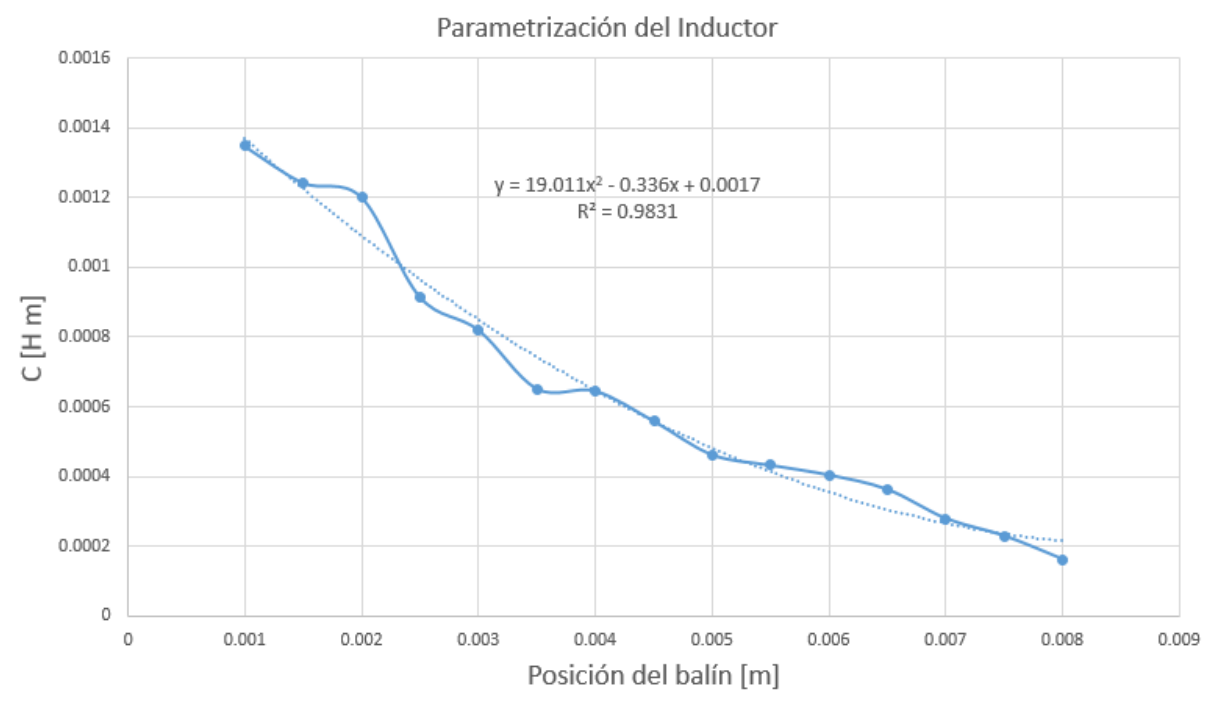

Figura 2: Resultado del experimento para determinar la constante $c$.

El experimento consistió en variar la posición del balín gradualmente desde 0.1 hasta $0.8 \mathrm{~cm}$ bajo el EI, en cada punto determinado llevar el sistema a su equilibrio $\left(\dot{x}_{1}=\dot{x}_{2}=\dot{x}_{3}=0\right.$ ), y cuando el equilibrio se lograra, medir dicha corriente. El resultado se puede observar en la Figura 2. La recta que mejor se ajusta a los datos experimentales es:

$$
c(x)=19,011 x_{1}^{2}-0,336 x_{1}+0,0017 ;
$$

Para determinar la función $G(u)$, bastó con medir la corriente en el EI a medida que se incrementaba sustancialmente la entrada al sistema $\mathrm{u}(\mathrm{t})$. Nótese que en la tercera ecuación de 8, cuando la corriente en el EI se estabiliza, es decir, que $\dot{x}_{3}=0$, necesariamente $G(u)=R x_{3}$. Los resultados se muestran en la figura 3 .

La Tabla 1 muestra en forma resumida los parámetros necesarios para linealizar el sistema alrededor del punto de operación.

El modelo matemático que representa la dinámica del sistema MAGLEV está dado por Ec. (8), este modelo es no lineal, a continuación se explica como linealizarlo alrededor del punto de operación $\left(\bar{x}_{1}, \bar{x}_{2}, \bar{x}_{3}, \bar{u}\right)$. 


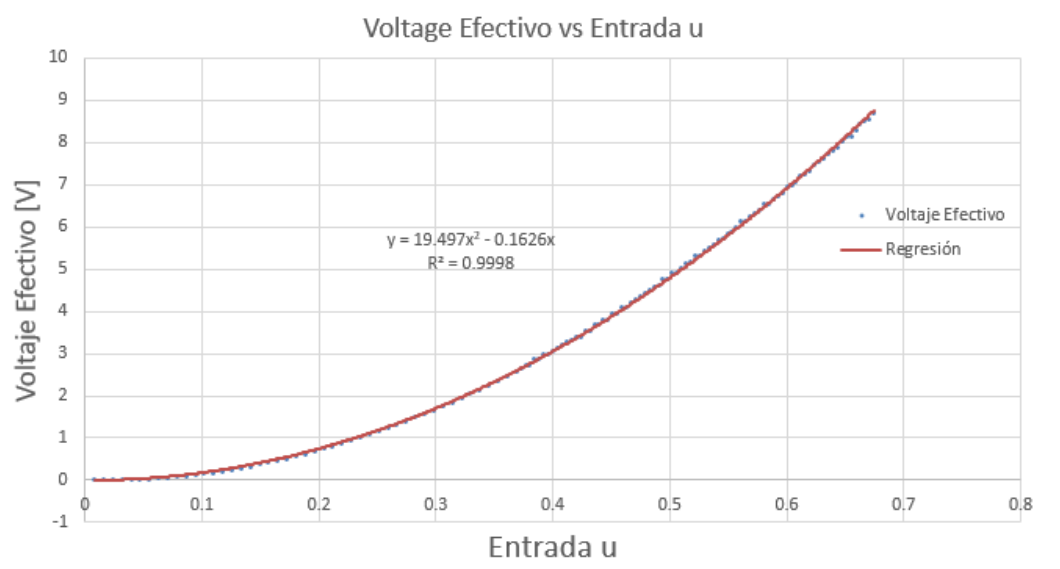

Figura 3: Resultado del experimento para determinar la $G(u)$ [Volts].

\begin{tabular}{|c|c|c|c|}
\hline Parámetro & Símbolo & Valor & Unidad \\
\hline Aceleración gravitacional & $g$ & 9,81 & $\mathrm{~m} / \mathrm{s}^{2}$ \\
Coeficiente de fricción viscosa & $\beta$ & $3,749 \times 10^{-6}$ & $\mathrm{~N} \cdot \mathrm{s} / \mathrm{m}$ \\
Masa de la esfera & $m$ & 0,0282 & $\mathrm{~kg}$ \\
Inductancia propia de la bobina & $L_{1}$ & 0,456 & $\mathrm{H}$ \\
Resistencia de la bobina & $R$ & 11,3 & $\Omega$ \\
\hline
\end{tabular}

Tabla 1: Parámetros del sistema de la planta MAGLEV

\subsection{Linealización del Modelo}

La linealización de modelos no lineales juega un papel importante en el diseño de controladores. El principio de linealización de ecuaciones no lineales, se basa en la suposición de que las variables del proceso cambian muy poco y sus desviaciones a partir del estado estable (estado estacionario) son pequeñas. La aproximación lineal puede calcularse usando la expansión en series de Taylor y considerando únicamente los términos lineales.

Consideremos un sistema dinámico no lineal de orden $n$ con $r$ entradas y $m$ salidas.

$$
\begin{aligned}
& \dot{x}(t)=f(x(t), u(t)) \quad \text { Ecuación de estado } \\
& y(t)=g(x(t), u(t)) \quad \text { Ecuación de salida }
\end{aligned}
$$

Donde:

- $x(t)=\left[x_{1}, \ldots, x_{n}\right]^{T}$ es el vector de estados del sistema. Las $n$ componentes del vector de estado, se llaman variables de estado, $x(t)$ determina unívocamente el estado del sistema**, especificada la entrada, para cualquier $t \geq t_{0}$.

- $u(t)=\left[u_{1}, \ldots, u_{r}\right]^{T}$ es el vector de entradas del sistema.

Definiendo $\bar{x}, \bar{u}$ tal que $f(\bar{x}, \bar{u})=0$ (Valores de estado estable), el modelo linealizado en espacio de estados es de la forma:

$$
\begin{aligned}
& \Delta \dot{x}(t)=A \Delta x(t)+B \Delta u(t) \\
& \Delta y(t)=C \Delta x(t)+D \Delta u(t)
\end{aligned}
$$

$* *$ El estado de un sistema es un conjunto mínimo de variables, tal que la información de estas variables y de las funciones de entrada, junto con las ecuaciones que describen la dinámica, proporcionan la salida y el estado futuro del sistema. 
Donde las variables de desviación son definidas como perturbaciones a partir de sus valores de estado estable (puntos de operación $* * *$ ).

$$
\begin{aligned}
\Delta x & =x-\bar{x} \\
\Delta u & =u-\bar{u} \\
\Delta y & =y-\bar{y}
\end{aligned}
$$

Los elementos de las matrices son definidos como:

$$
\begin{array}{rlrl}
A_{i j} & =\left.\frac{\partial f_{i}}{\partial x_{j}}\right|_{\bar{x}, \bar{u}} & B_{i j} & =\left.\frac{\partial f_{i}}{\partial u_{j}}\right|_{\bar{x}, \bar{u}} \\
C_{i j} & =\left.\frac{\partial g_{i}}{\partial x_{j}}\right|_{\bar{x}, \bar{u}} D_{i j}=\left.\frac{\partial g_{i}}{\partial u_{j}}\right|_{\bar{x}, \bar{u}}
\end{array}
$$

Los subíndices $i j$ se refieren a la $i$-ésima fila y j-ésima columna de la matriz correspondiente. Por ejemplo, el elemento $B_{i j}$ relaciona el efecto de la $j$-ésima entrada sobre la $i$-ésima derivada del estado.

El punto de equilibrio escogido para realizar la linealización es:

$$
\begin{aligned}
\bar{x}_{1} & =0,005 & \bar{x}_{2} & =0 \\
\bar{x}_{3} & =0,235 & \bar{u} & =2,56
\end{aligned}
$$

Las matrices en espacio de estados que definen el comportamiento dinámico de la planta se muestran en la Ec. (14)

$$
\begin{aligned}
& A=\left[\begin{array}{ccc}
0 & 1 & 0 \\
\frac{2 C x_{3}^{2}}{m x_{1}} & -\frac{\beta}{m} & -\frac{2 C x_{3}}{m x_{1}^{2}} \\
\frac{2 C\left(u-R x_{3}+\frac{2 C x_{2} x_{3}}{x_{1}{ }^{2}}\right)}{x_{1}{ }^{2}\left(L_{1}+\frac{2 C}{x_{1}}\right)^{2}}-\frac{4 C x_{2} x_{3}}{x_{1}{ }^{3}\left(L_{1}+\frac{2 C}{x_{1}}\right)} & \frac{2 C x_{3}}{x_{1}{ }^{2}\left(L_{1}+\frac{2 C}{x_{1}}\right)} & -\frac{R-\frac{2 C x_{2}}{x_{1}{ }^{2}}}{L_{1}+\frac{2 C}{x_{1}}}
\end{array}\right] \\
& B=\left[\begin{array}{c}
0 \\
0 \\
\frac{1}{L_{1}+\frac{2 C}{x_{1}}}
\end{array}\right]_{\bar{x}, \bar{u}} \quad C=\left[\begin{array}{lll}
100 & 0 & 0
\end{array}\right] \quad D=\left[\begin{array}{l}
0 \\
0 \\
0
\end{array}\right]
\end{aligned}
$$

Con las matrices $A, B, C$ y $D$ anteriores se obtiene una representación lineal en espacio de estados del sistema MAGLEV alrededor del punto de equilibrio definido por Ec. (13).

Para obtener la función de transferencia que representa el modelo lineal, utilizamos la expresión, [13]:

$$
G(s)=C(s \mathbf{I}-A)^{-1} B+D
$$

Con los valores numéricos de la Tabla 1, se obtiene la función de transferencia del sistema:

$$
G(s)=\frac{-4,045 * 10^{5}}{(s-74,66)(s+57,13)(s+47,22)}
$$

La función de transferencia de la Ec. (16) tiene tres polos, uno de ellos con parte real positiva, lo que hace que al sistema inestable en lazo abierto, una forma de estabilizarlo es implementar un control PID como se explica en la siguiente sección.

* *mbíen llamados puntos de equilibrio. 


\section{Control PID}

La ley de control de un PID en su forma "académica", [13] se escribe:

$$
u(t)=k_{p} e(t)+\frac{k_{p}}{T_{i}} \int_{0}^{t} e(t) d t+k_{p} T_{d} \frac{d e(t)}{d t}
$$

Donde $u$ es la señal de control, $e$ es la señal de error, $k_{p}$ es la ganancia proporcional, $T_{i}$ es la constante de tiempo integral y $T_{d}$ es la constante de tiempo derivativa. La señal de control es la suma de tres términos: la acción proporcional $(\mathbf{P})$ que corresponde a un control proporcional, la acción integral (I) da una acción de control que es proporcional a la integral del error y la acción derivativa $(\mathbf{D})$ es proporcional a la variación temporal de la señal de error.

\subsection{Simulación y Sintonización del PD}

El controlador PD paralelo fue sintonizado con ayuda de la app PID Tuner de MATLAB. Los valores de las constantes del controlador se muestran en la Tabla 2 .

\begin{tabular}{|c|c|}
\hline Offset & 0 \\
\hline$K_{p}$ & -3.35 \\
\hline$T_{d}$ & 0.1354 \\
\hline
\end{tabular}

Tabla 2: Constantes de un controlador PD.

Una vez obtenidas estas constantes, el algoritmo de control PID se implementó en un controlador digital haciendo uso de la tarjeta Arduino, esta tarjeta tiene entradas y salidas analógicas y digitales y es de fácil y libre programación, lo que la hace ideal para proyectos de instrumentación y control, [10. El resultado de la implementación del controlador, Ec. (17) es que el sistema sigue siendo inestable. La sintonización del controlador no fue la adecuada, debido a que el modelo no considera todas las no-linealidades. Es preciso anotar que en el modelo del sistema no se considera la saturación de la acción de control, ya que no es capaz de producir voltajes negativos ni mayores a 5 [Volts], pues la tarjeta Arduino entrega voltajes entre 0 y 5 [Volts]. La ley de control normalizada entrega valores entre 0 y 1 . No se considera el espacio de libertad limitado del balín, pues no puede estar en posiciones menores que $0 \mathrm{~mm}$ ni mayores a $8 \mathrm{~mm},\left(0 \leq X_{0} \leq 8 \mathrm{~mm}\right)$.

Con ayuda de Simulink, se realizó una simulación donde se tiene en cuenta los límites en las variables ya mencionadas. En la figura 4 se muestra el diagrama de bloques implementado. En él se puede notar el bloque de S saturación de la señal de control. Las ecuaciones de estado del sistema No-lineal son implementadas en el bloque "Sistema no-lineal" que es una S-function. En el código de dicha $S$-function, se programó una fuerza de impacto que actuaría para posiciones menores a $1 \mathrm{~mm}$ y mayores a $8 \mathrm{~mm}$ (Cabe señalar que en las ecuaciones de estado, $x_{1}$ debe ser diferente de cero). Se sintonizó el controlador PD para este nuevo modelo del sistema y se obtuvieron las constantes que se muestran en la Tabla 3 .

\begin{tabular}{|c|c|}
\hline Offset & 0.291 \\
\hline$K_{p}$ & -0.58 \\
\hline$T_{d}$ & 0.05 \\
\hline
\end{tabular}

Tabla 3: Constantes de un controlador PD, Experimento 2. 


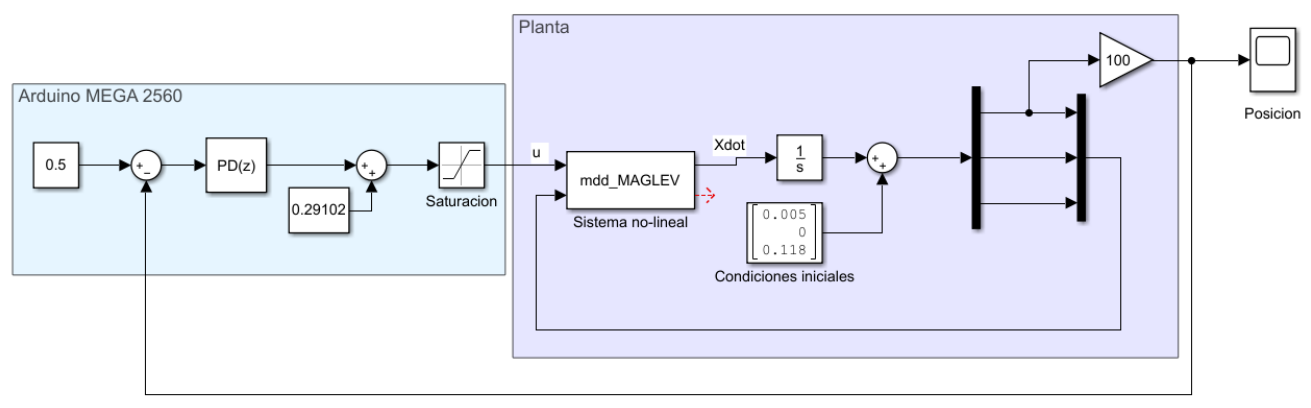

Figura 4: Diagrama de bloques implementado en Simulink del controlador PD

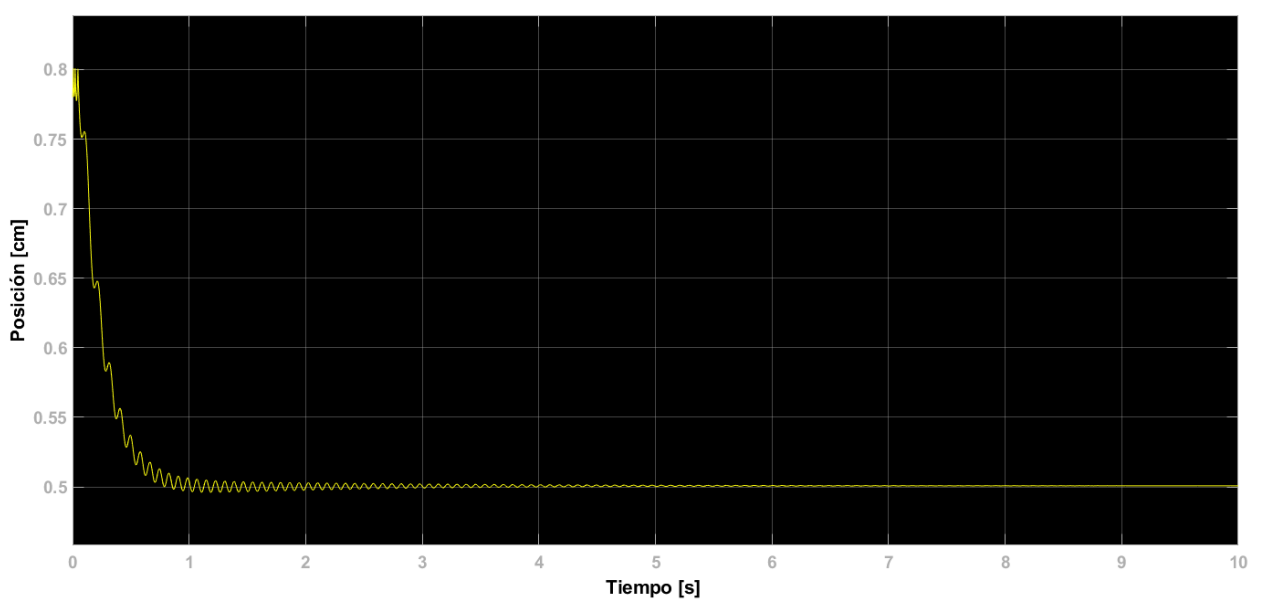

Figura 5: Resultado de la simulación del controlador PD.

La figura 5 muestra el resultado de la simulación del sistema durante 10 segundos. La posición inicial es $0.8 \mathrm{~cm}$ y la referencia a seguir es $0.5 \mathrm{~cm}$.

La ecuación característica de la planta muestra que el sistema tiene tres polos, uno de ellos inestable, por lo que el controlador a implementar sólo debe contener acciones proporcional y derivativa, para así eliminar el polo inestable del sistema. La sintonización, se realizó entonces por ensayo y error de las constantes $K_{p}$ y $T_{d}$.

El parámetro $u_{0}$ se refiere al valor del Offset, es decir, la entrada a la planta necesaria para que el sistema esté en equilibrio. Este parámetro se encontró experimentalmente: En lazo abierto, se ubicó el balín en la posición que sería a la cual se desea hacer levitar (concretamente, a una distancia de $0.5 \mathrm{~cm}$ bajo el EI), se incrementó gradualmente la acción de control (entre 0 y 1 ), hasta ocasionar que el balín se elevara, atraído por la fuerza del electroimán. El valor encontrado fue 0.295 .

Para determinar el parámetro de $K_{p}$ se realizó el mismo procedimiento anterior, esta vez para diferentes distancias, en una secuencia desde $0.05 \mathrm{~cm}$ hasta $0.8 \mathrm{~cm}$ con escala de $0.05 \mathrm{~cm}$. Los resultados se muestran en la figura 6. Con ayuda de MATLAB, se hizo una regresión lineal para encontrar una recta que se ajustara a los datos obtenidos. Como resultado, se obtuvo que dicha recta tiene como función: 


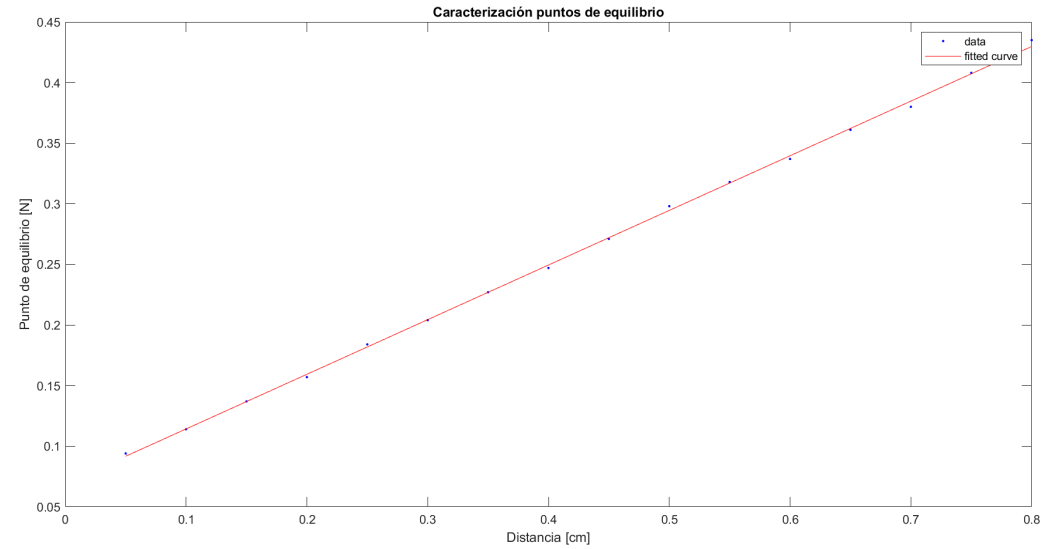

Figura 6: Ley de control de equilibrio normalizada para distintas distancias.

$$
u(x)=0,4507 x+0,0692
$$

donde $x$ es la distancia, es decir, la salida del sistema. Como la ley de control debe estar en función del error y no de la Salida del sistema, reemplazamos en la función anterior la relación que define el error: $e=r-x$, donde $r$ es la señal de referencia. Se obtiene:

$$
u(e, r)=0,4507(r-e)+0,0692
$$

Para el caso particular cuando la referencia es $0.5 \mathrm{~cm}$, la Ec. (19) se vuelve:

$$
u(e)=-0,4507 e+0,2945
$$

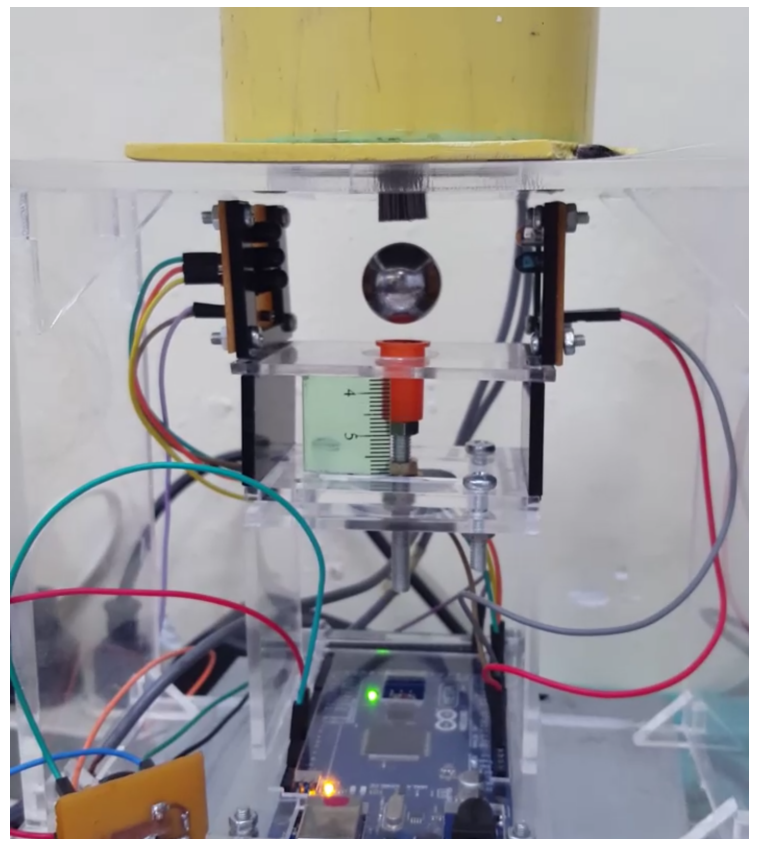

Figura 7: Fotografía del prototipo MAGLEV, resultado de la sintonización del controlador PD. 


\begin{tabular}{|c|c|}
\hline Offset & 0.295 \\
\hline$K_{p}$ & -0.643 \\
\hline$T_{d}$ & 0.079 \\
\hline
\end{tabular}

Tabla 4: Constantes de un controlador PD.

La Ec. 20 se tomó como punto de partida para la sintonización del controlador $P D$ a implementar. Por ensayo y error, variando el Offset, $K_{p}$ y $T_{d}$ se logró estabilizar el sistema como lo muestra la figura 7. Las constantes que resultaron estabilizar el sistema se muestran en la Tabla 4

\section{Resultados}

En la tarjeta Arduino Mega 2560 se registraron los datos de la salida del sistema por un lapso de tiempo de 12 segundos y se obtuvo la respuesta que se muestra en la figura 8. Los siguientes índices de desempeño se utilizaron para validar estadísticamente el controlador PD:

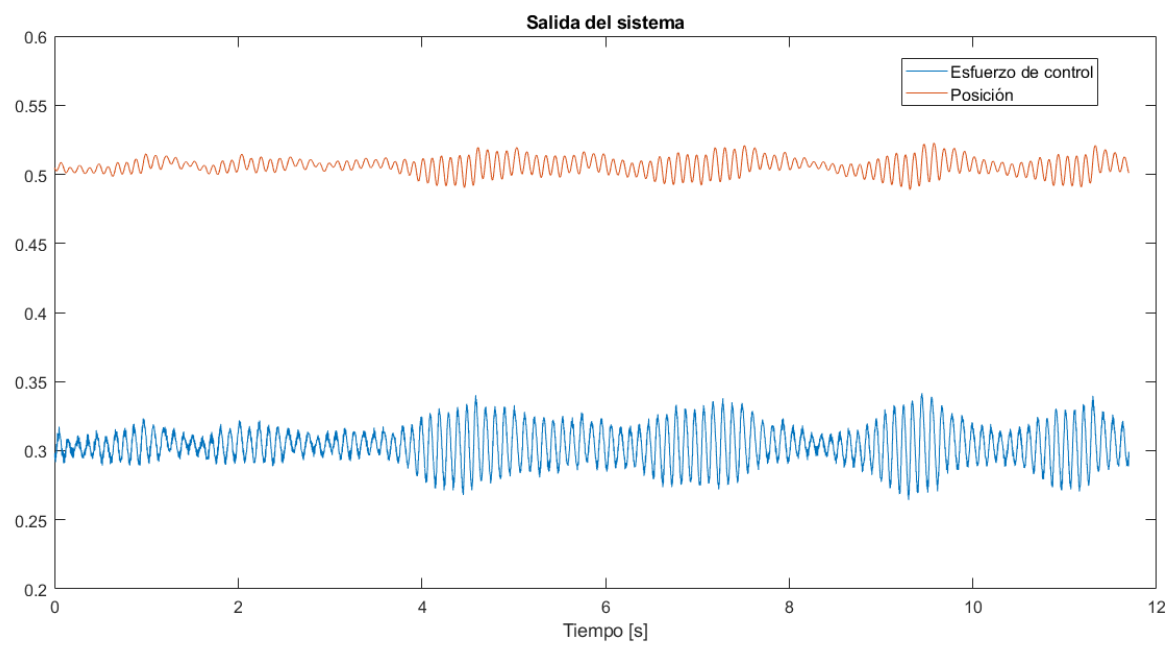

Figura 8: Salida del sistema MAGLEV con un controlador PD.

\section{Desviación estándar del error:}

$$
\epsilon_{e}=\sqrt{\frac{1}{N} \sum_{k=1}^{N}\left[x_{d}(k)-x(k)\right]^{2}}
$$

$\epsilon_{e}$ es la desviación estándar del error, $\mathrm{N}$ el número de muestras, $x_{d}(k)$ es el valor de posición deseado y $x(k)$ es el valor de posición medido.

Valor RMS del esfuerzo de control:

$$
\epsilon_{u}=\sqrt{\frac{1}{N} \sum_{k=1}^{N}[u(k)]^{2}}
$$

Para este caso $\epsilon_{u}$ es el valor RMS del esfuerzo de control $u(k)$. 


\begin{tabular}{|c|c|c|}
\hline No. & $\epsilon_{e}$ & $\epsilon_{u}$ \\
\hline 1 & 0.000252 & 0.09231 \\
2 & 0.000295 & 0.09295 \\
3 & 0.000320 & 0.09298 \\
4 & 0.000444 & 0.09293 \\
5 & 0.000373 & 0.09120 \\
6 & 0.000487 & 0.09250 \\
7 & 0.000313 & 0.09278 \\
8 & 0.000453 & 0.09245 \\
9 & 0.000297 & 0.09273 \\
10 & 0.000273 & 0.09228 \\
\hline Promedio & 0.000351 & 0.09251 \\
\hline
\end{tabular}

Tabla 5: Índices de desempeño del controlador PD en la planta MAGLEV.

En la Tabla 5 se muestra el valor promedio de los índices de desempeño de 10 experimentos. El número total de datos para cada uno de los experimentos es $N=3900$, para un valor de posición deseado de $x_{d}(k)=0,5$.

Los índices de desempeño muestran que el sistema fue estabilizado satisfactoriamente alrededor de un punto de equilibrio, con una desviación estándar del error de 0,035\%.

\section{Conclusiones}

Se diseñó y se construyó una planta de Levitación Magnética de bajo costo que puede ser utilizada para la experimentación en sistemas de control por los estudiantes del programa de Ingeniería Física de la Universidad del Cauca. La planta de levitación magnética le permite a los estudiantes, además de aplicar los conocimientos adquiridos en su curso de Sistemas de Control, enfrentarse con problemas reales tales como: Incompatibilidad modelo-sistema real, desconocimiento de parámetros del sistema, ruido de sensado, disturbios externos en la planta y limitaciones en la acción de control.

El costo total de construcción de la planta fue $\$ 272.000 \mathrm{COP}$, lo que se considera un bajo costo comparado con otras plantas de levitación magnética similares que se encuentran en el mercado.

\section{Agradecimientos}

Los autores de este artículo expresan sus más sinceros agradecimientos a la Universidad del Cauca por todo el apoyo que les fue dado en la realización del proyecto.

\section{Referencias}

[1] J. Meins, L. Miller, and W. J. Mayer, "The high speed maglev transport system transrapid," IEEE Transactions on Magnetics, vol. 24, no. 2, pp. 808-811, March 1988.

[2] A. J. Ellison and H. Bahmanyar, "Surface-guided transport systems of the future," Proceedings of the Institution of Electrical Engineers, vol. 121, no. 11, pp. 1224-1248, November 1974.

[3] W. Leonhard, R. G. Rhodes, and J. H. Rakels, "Technical alternatives for a maglev system," Electronics and Power, vol. 24, no. 4, pp. 293-296, April 1978.

[4] B. V. Jayawant, P. K. Sinha, A. R. Wheeler, R. J. Whorlow, and J. Willsher, "Development of 1-ton magnetically suspended vehicle using controlled d.c. electromagnets," Proceedings of the Institution of Electrical Engineers, vol. 123, no. 9, pp. 941-948, Sep. 1976.

[5] T. H. Wong, "Design of a magnetic levitation control system -an undergraduate project," IEEE Transactions on Education, vol. E-29, no. 4, pp. 196-200, Nov 1986.

[6] W. Barie and J. Chiasson, "Linear and nonlinear state-space controllers for magnetic levitation," International Journal of Systems Science - IJSySc, vol. 27, pp. 1153-1163, 111996. 
[7] N. Sotnikova, M. Zhabko and T. Lepikhin, "Control systems analysis and design labs with educational plants," IFAC Proceedings Volumes, vol. 45, no. 11, pp. 212 - 217, 2012, 9th IFAC Symposium Advances in Control Education. [Online]. Available: http://www.sciencedirect.com/science/article/pii/S1474667015376059

[8] W. Yu and X. Li, "A magnetic levitation system for advanced control education," IFAC Proceedings Volumes, vol. 47, no. 3, pp. 9032 - 9037, 2014, 19th IFAC World Congress. [Online]. Available: http://www.sciencedirect.com/science/article/pii/S1474667016430399

[9] J. I. Artigas, L. A. Barragan, S. Llorente, A. Marco, and O. Lucia, "Low-cost magnetic levitation system for electronics learning," in 2010 4th IEEE International Conference on E-Learning in Industrial Electronics, 2010, pp. 55-60.

[10] C. F. Rengifo and D. A. Bravo, "A project-based learning approach to teach identification and control systems," IEEE Revista Iberoamericana de Tecnologias del Aprendizaje, vol. 15, no. 1, pp. 10-16, 2020.

[11] F. Golnaraghi and B. C. Kuo, Automatic control systems, 9th ed. Wiley, 2009.

[12] L. Landau and E. Lifshitz, Mecánica, ser. Curso de física teórica. Reverté, 1978, no. v. 1.

[13] K. Åström and T. Hägglund, Advanced PID Control. ISA-The Instrumentation, Systems, and Automation Society, 2006.
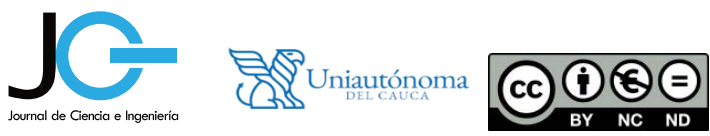